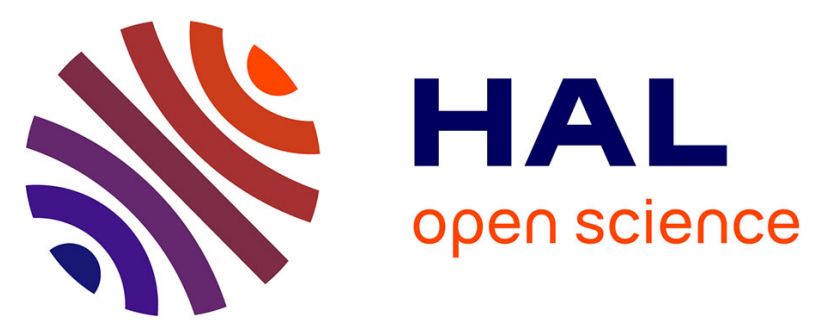

\title{
Influence de la non-stœchiométrie et de l'addition de vanadium sur l'état d'ordre et les propriétés mécaniques d'alliages fer-cobalt
}

\author{
J.L. Demenet, J.P. Eymery, P. Moine
}

\section{> To cite this version:}

J.L. Demenet, J.P. Eymery, P. Moine. Influence de la non-stœehiométrie et de l'addition de vanadium sur l'état d'ordre et les propriétés mécaniques d'alliages fer-cobalt. Revue de Physique Appliquée, 1974, 9 (4), pp.721-729. 10.1051/rphysap:0197400904072100 . jpa-00243839

HAL Id: jpa-00243839

https://hal.science/jpa-00243839

Submitted on 1 Jan 1974

HAL is a multi-disciplinary open access archive for the deposit and dissemination of scientific research documents, whether they are published or not. The documents may come from teaching and research institutions in France or abroad, or from public or private research centers.
L'archive ouverte pluridisciplinaire HAL, est destinée au dépôt et à la diffusion de documents scientifiques de niveau recherche, publiés ou non, émanant des établissements d'enseignement et de recherche français ou étrangers, des laboratoires publics ou privés. 
Classification

Physics Abstracts

7.222

\title{
INFLUENCE DE LA NON-STCECHIOMÉTRIE \\ ET DE L'ADDITION DE VANADIUM SUR L'ÉTAT D'ORDRE ET LES PROPRIÉTÉS MÉCANIQUES D'ALLIAGES FER-COBALT
}

\author{
J. L. DEMENET, J. P. EYMERY et P. MOINE \\ Laboratoire de Métallurgie Physique (*) \\ 40, avenue du Recteur-Pineau, 86022 Poitiers, France
}

(Reçu le 2 janvier 1974, révisé le 29 janvier 1974)

\begin{abstract}
Résumé. - On étudie l'influence de la non-stœchiométrie et de l'addition de vanadium sur quelques aspects des problèmes posés par la détermination de l'état d'ordre et des propriétés mécaniques des alliages $\mathrm{Fe}-\mathrm{Co}$. On a observé des domaines d'ordre après recuit et mesuré le degré d'ordre à longue distance en fonction de la température de trempe. Dans le domaine des propriétés mécaniques, on a constaté que le maximum de limite élastique a lieu sur les différents alliages, pour un degré d'ordre à longue distance d'environ 0,55 , ce qui correspond à une distance théorique des partielles d'une superdislocation de l'ordre de $500 \AA$. Une transition fragile-ductile est observée en même temps. Cette valeur de $500 \AA$ semble liée au moment où les partielles peuvent effectuer des glissements déviés indépendamment l'une de l'autre.
\end{abstract}

\begin{abstract}
Non-stoichiometry and vanadium-addition influence on the order state and mechanical properties of various $\mathrm{Fe}-\mathrm{Co}$ alloys is studied. Ordered domains have been observed after annealing and the long range order parameter $S$ determined as a function of quenching temperature. Moreover a peak in yield stress has been observed for each alloy for $S \# 0.55$ which corresponds to a superdislocation spacing of about $500 \AA$. A brittle to ductile transition is also observed at the same time. The value of $500 \AA$ seems to be connected to the cross slipping ability for the partials of the superdislocations.
\end{abstract}

1. Introduction. - La déformation plastique et la fragilité des alliages fer-cobalt en fonction de l'état d'ordre (type L20) ont fait l'objet de nombreuses recherches. Dans le domaine des composés binaires les principaux travaux sont dus à Stanley [1], Facaros et Trap [2], Marcinkowski et Chessin [3], Chen [4], Marcinkowski et Fisher [5], Marcinkowski et Larsen [6], Sheng-Ti-Fong et al. [7]. En ce qui concerne l'alliage ternaire $\mathrm{Fe}-\mathrm{Co}-2 \mathrm{~V}(49 \%$ en poids $\mathrm{Fe}, 49 \%$ en poids Co $2 \%$ en poids V), il faut citer Chen et Wiener [8], Chen [9], Stoloff et Davies [10], Johnston et al. [11], Jordan et Stoloff [12, 13], Koylu et al. [14], Moine et al [15], Dinhut et al. [16], Bonou [17].

Dans cet article nous nous intéressons à l'influence de la non-stœchiométrie et au rôle de l'addition de vanadium sur quelques aspects des problèmes posés par la détermination de l'état d'ordre et des propriétés mécaniques des alliages $\mathrm{Fe}-\mathrm{Co}$. L'étude a porté sur les compositions pondérales suivantes : 30, 40, 50, 60 et $70 \%$ de cobalt (notées par la suite, respectivement Fe-Co 70-30, Fe-Co 60-40, Fe-Co 50-50, Fe- ̃u 40-60

$\left(^{*}\right)$ Associé au C. N. R. S. et $\mathrm{Fe}-\mathrm{Co}$ 30-70), et a été réalisée par diffraction des rayons $\mathrm{X}$, microscopie électronique et essais de traction.

2. Méthodes expérimentales. - Le dispositif de diffraction des rayons $X$ est un montage classique par réflexion, avec la particularité suivante : pour minimiser les effets de texture, on utilise un porte-échantillon tournant, sous vide. Ce dispositif a déjà été décrit par Eymery et al. [18].

Le paramètre d'ordre à longue distance $S$ mesuré par diffraction des rayons X est, d'après Warren [19], égal à :

$$
S=\frac{r_{\alpha}-F_{\mathrm{A}}}{1-F_{\alpha}} \quad\left(S \text { proportionnel à } r_{\alpha}+r_{\beta}\right)
$$

avec :

$r_{\alpha}$ : fraction de sites $\alpha$ occupés par un atome $\mathrm{A}$, $r_{\beta}$ : fraction de sites $\beta$ occupés par un atome $\mathbf{B}$, $F_{\mathrm{A}}$ : fraction d'atomes $\mathrm{A}$, $F_{\alpha}:$ fraction de sites $\alpha$.

La relation entre ce paramètre et le paramètre $S_{0}$ 
généralisé de Bragg et Williams $\left(S_{0}\right.$ proportionnel à $\left.r_{\alpha}\right)$

$$
S_{0}=\frac{r_{\alpha}-F_{\mathrm{A}}}{\frac{F_{\mathrm{A}}}{F_{\alpha}}-F_{\mathrm{A}}}, \quad \text { est la suivante : } \quad S=\frac{F_{\mathrm{A}}}{F_{\alpha}} S_{0} .
$$

Les essais de traction sont effectués sur une machine Instron. La vitesse de déformation est de $0,02 \mathrm{~cm} / \mathrm{min}$. Les éprouvettes de traction, de forme cylindrique, ont une longueur utile de $8 \mathrm{~mm}$, un diamètre de $3 \mathrm{~mm}$, et les congès de raccordement ont $1 \mathrm{~mm}$ de rayon. Les microfissures dues à l'usinage sont éliminées par polissage électrolytique.

Les principaux résultats fournis par les courbes effort-déformation sont les suivants :

- la limite élastique macroscopique $\sigma_{\mathrm{E}}$,

- la charge à la rupture $\sigma_{\mathrm{R}}$,

- la striction $\Sigma \%$ définie par :

$$
\Sigma \%=\frac{S_{0}-S_{\mathrm{f}}}{S_{0}} 100
$$

$S_{0}$ et $S_{\mathrm{f}}$ étant les sections de l'éprouvette avant et après l'essai

— l'allongement à la rupture $A \%$ défini par :

$$
A \%=\frac{L_{\mathrm{f}}-L_{0}}{L_{0}} 100
$$

$L_{0}$ et $L_{\mathrm{f}}$ étant les longueurs de l'éprouvette avant et après la traction.

Les deux derniers paramètres $A \%$ et $\Sigma \%$ ne sont pas équivalents : l'éprouvette s'allonge pendant l'essai, alors que la striction n'apparaît qu'en fin de déformation.

L'état d'ordre maximum est obtenu, quelle que soit la concentration en cobalt, par recuit $1 \mathrm{~h}$ à $900^{\circ} \mathrm{C}$, sous vide secondaire, suivi d'un refroidissement lent à la vitesse d'environ $70 \% / \mathrm{h}$.

L'état désordonné est obtenu par trempe à l'eau au-dessus de $T_{\mathrm{C}}$ (température critique de transformation ordre-désordre).

3. Etat structural. - L'état structural est étudié après recuit et après trempe en nous limitant, pour ce dernier cas, aux alliages Fe-Co 50-50 et Fe-Co 40-60.

3.1. - Etat STRUCtURAL APRÈs ReCUit. - Des domaines antiphases ont déjà été observés dans le cas $\mathrm{du}$ système fer-cobalt, sur le composé $\mathrm{Fe}-\mathrm{Co}-2 \mathrm{~V}$ par Krahl [20] et Dinhut et al. [16] (Fig. 1a). Nous avons observé de tels domaines dans les alliages binaires $\mathrm{Fe}-\mathrm{Co}$ de différentes concentrations 60, 50, $40,30 \%$ en poids de fer.

La figure $1 b$ montre une image en fond noir des domaines antiphases dans un échantillon $\mathrm{Fe}-\mathrm{Co}$ 50-50 ; l'image a été prise avec une réflexion (001), le plan normal au faisceau électronique est du type (120). La forme des domaines est sensiblement la même que dans le Fe-Co-2 V, et correspond à la structure
" en gruyère ", prévue théoriquement par English (1966) [21]. La taille moyenne des domaines, déterminée par la méthode des intersections, est de l'ordre de $3700 \AA \AA$. Des observations analogues ont été réalisées sur des échantillons de Fe-Co 40-60 (Fig. 1c)

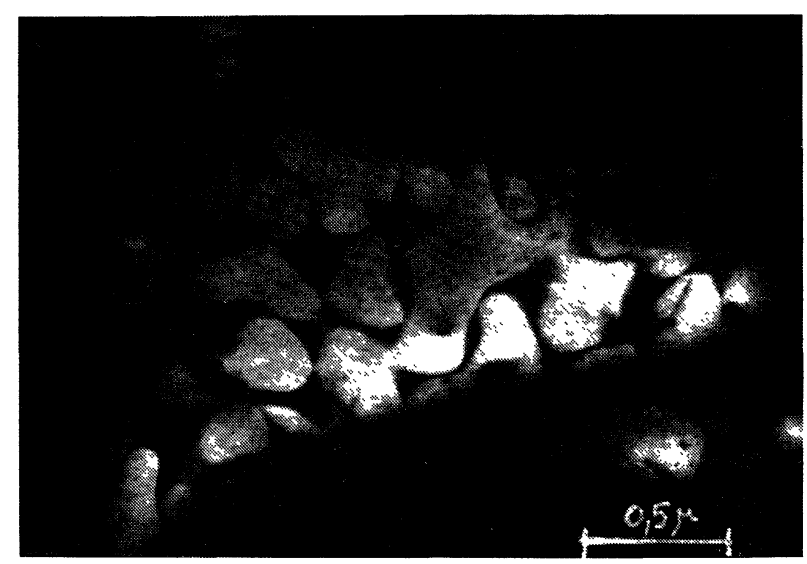

FIG. 1a. - Domaines antiphases dans l'alliage Fe-Co-2 V $(\times 29000) g=(100)$.

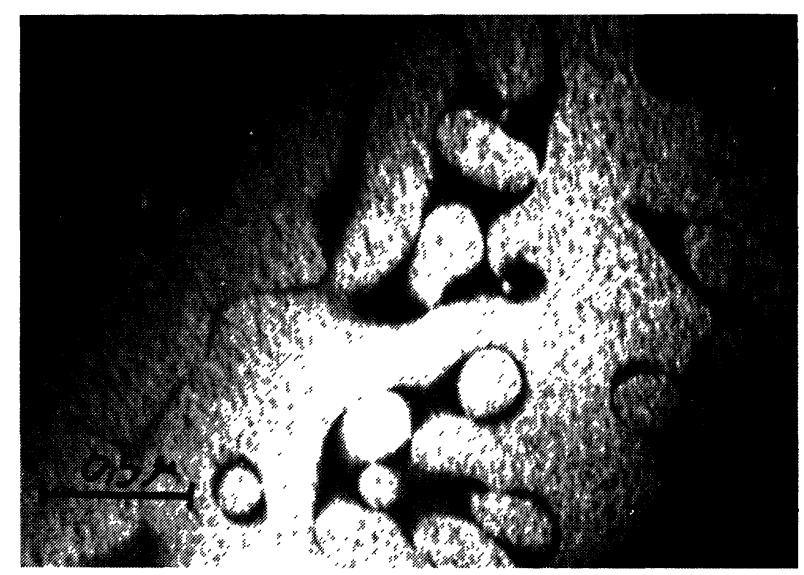

FIG. 1b. - Domaines antiphases dans l'alliage Fe-Co 50-50 $(\times 29000 \mathrm{~g}=(001)$.

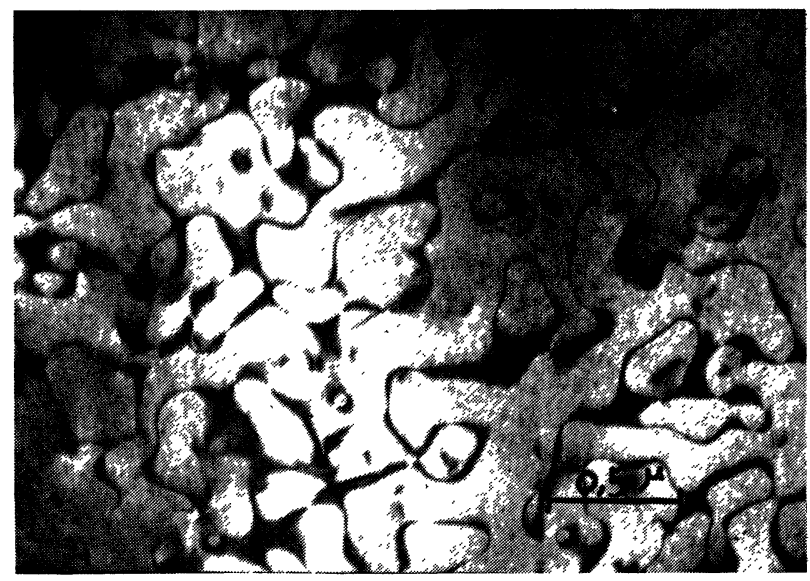

Fig. 1c. - Domaines antiphases dans l'alliage Fe-Co 40-60 $(\times 26500) g=(100)$. 


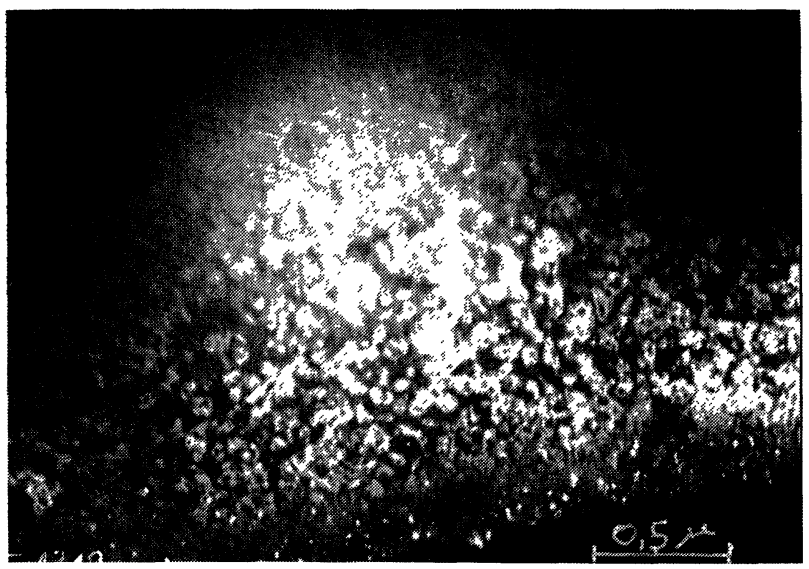

Fig. 1d. - Domaines antiphases dans l'alliage Fe-Co $60-40$ $(\times 27000) g=(001)$.

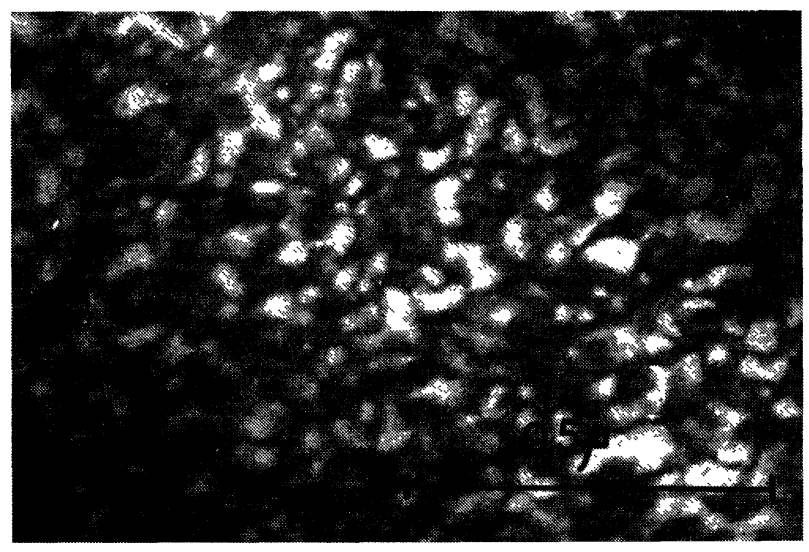

FIG. 1e. - Domaines antiphases dans l'alliage Fe-Co 30-70 $(\times 73500) g=(100)$.

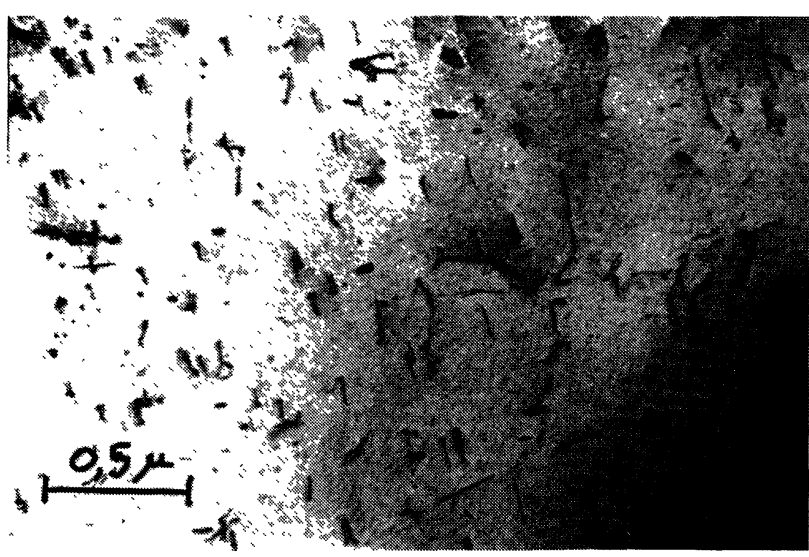

FIG. 1f: - Dislocations doubles dans l'alliage Fe-Co 70-30

$$
(\times 29000) g=(200) \text {. }
$$

et de Fe-Co 60-40 (Fig. 1d). Les domaines ont un diamètre moyen de $2500 \AA$ dans le cas de l'alliage Fe-Co 40-60 et de $1700 \AA$ dans le cas de l'alliage $\mathrm{Fe}-\mathrm{Co}$ 60-40.

D'après les diagrammes d'équilibre les plus complets (Hansen et Anderko [22], Ellis et Greiner [23]), la composition $\mathrm{Fe}-\mathrm{Co}$ 30-70 correspond à la valeur extrême en fer au-dessous de laquelle l'alliage n'est plus ordonné ; le paramètre d'ordre à longue distance mesurable par diffraction des rayons $X$ ne peut pas dépasser 0,6. Pour notre part, nous avons observé aux rayons $\mathrm{X}$ des raies de structure, et par microscopie électronique des images faiblement contrastées de domaines antiphases (Fig. 1e). Les domaines sont petits, environ $500 \AA$, et difficiles à observer du fait de la faible intensité dis réflexions de surstructure.

Par contre l'étude de l'alliage Fe-Co 70-30, tant par diffraction des rayons $\mathrm{X}$ que par diffraction électronique, ne nous a pas permis de mettre en évidence de surstructure. Néanmoins l'existence de dislocations appariées (Fig. 1f) semble prouver qu'il existe de l'ordre à courte distance, et peut-être même un ordre à longue distance très faible $(S<0,25)$.

Pour toutes ces observations, le contraste des frontières ne présente pas de franges. Cela est probablement dû au fait que la distance d'extinction des réflexions de surstructure est comparable à l'épaisseur des échantillons.

3.2 EtAT STRUCTURAL APRÈS TREMPE. - Nous nous sommes limités, pour cette étude, au cas des alliages Fe-Co 50-50 et Fe-Co 40-60. A l'aide du diffractomètre $X$, nous avons déterminé les courbes $S=f\left(T_{\mathrm{t}}\right)$, où $T_{\mathrm{t}}$ est la température de trempe, et $S$ le degré d'ordre à longue distance défini par Warren. Les échantillons, initialement ordonnés, sont portés pendant vingt minutes à la température désirée, puis trempés à l'eau. Les résultats sont rapportés sur les figures 2 fet 3 . A côté des courbes expérimentales, nous avons tracé sur le même graphique, les courbes théoriques de Bragg et Williams $S=f(T)$, où $T$ représente la température d'essai (voir [24] pour l'obtention des courbes $S=f(T)$ ). La figure 2 montre une similitude entre le Fe-Co 50-50 et le Fe-Co-2 V,

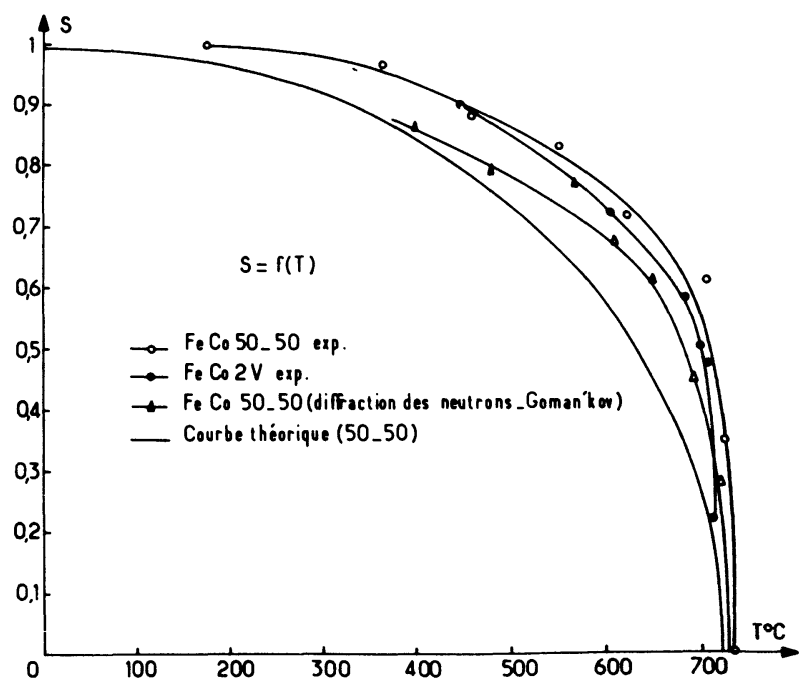

Fig. 2. - Evolution du degré d'ordre $\mathrm{S}$ en fonction de la température dans le cas de l'alliage équiatomique. Courbe théorique de Bragg et Williams et courbes expérimentales. 


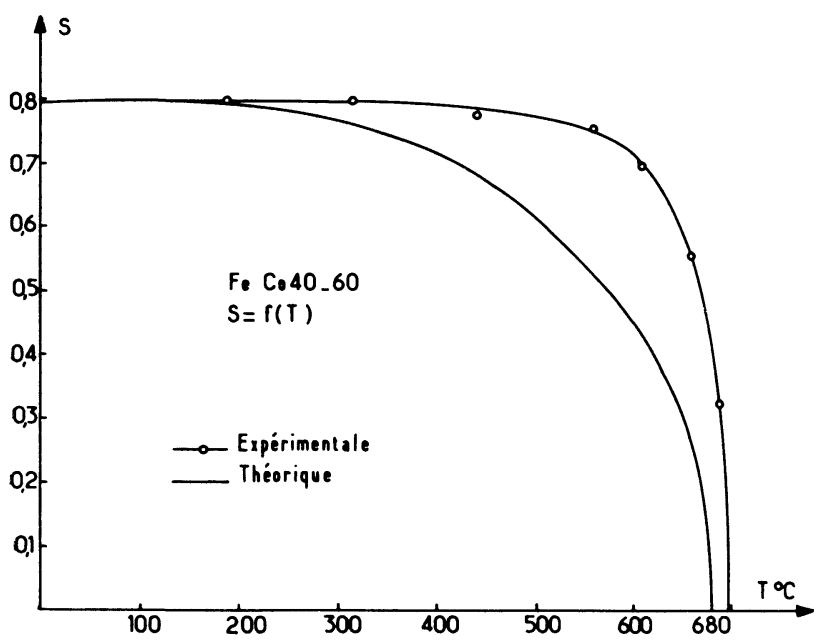

FIG. 3. - Evolution du degré d'ordre $S$ en fonction de la température dans le cas de l'alliage Fe-Co 40-60. Courbe théorique et courbe expérimentale.

ainsi qu'un faible écart entre les courbes expérimentales et la courbe théorique, aisément explicable puisqu'au cours d'une trempe à l'eau, même rapide, il y a toujours une légère remise en ordre. Sur la même figure, nous avons tracé la courbe relative au Fe-Co 5050 obtenue par Goman'kov [25] par diffraction des neutrons: on constate une assez bonne similitude des résultats.

En ce qui concerne l'alliage $\mathrm{Fe}-\mathrm{Co} 40-60$, les résultats (Fig. 3) montrent une différence notable entre les deux courbes, théorique $(S=f(T))$ et expérimentale $\left(S=f\left(T_{\mathrm{t}}\right)\right)$; cet alliage semble se mettre en ordre pendant la trempe, plus rapidement que l'alliage équiatomique.

D'autre part, pour les trois concentrations, nous n'avons pas constaté d'élargissement de la raie (100) après trempe, ce qui indique que les domaines antiphases gardent la taille moyenne qu'ils ont à l'état recuit, tant que la température est inférieure à $T_{\mathrm{C}}$.

L'accélération de la remise en ordre lorsque la stœchiométrie n'est pas respectée, peut être expliquée en considérant que l'énergie de migration des lacunes est plus importante dans l'alliage $\mathrm{Fe}-\mathrm{Co}$ 50-50 que dans l'alliage $\mathrm{Fe}-\mathrm{Co}$ 40-60. En effet d'après Girifalco [26] l'énergie de migration est de la forme : $E_{\mathrm{m}}=E_{0}+A S^{2}$, et la vitesse de migration :

$$
v=K \exp \left(-\frac{E_{\mathrm{m}}}{k T_{\mathrm{t}}}\right) \quad\left(E_{0}, A \text { et } K \text { constants }\right) \text {. }
$$

Or le Fe-Co 40-60 a un degré d'ordre maximum de 0,8 alors que le Fe-Co 50-50 a un degré d'ordre maximum égal à 1 . Donc l'énergie de migration des lacunes dans le Fe-Co 40-60 est inférieure à celle des lacunes dans le Fe-Co 50-50 ; et il s'ensuit que la vitesse de migration des lacunes est plus faible dans le $\mathrm{Fe}-\mathrm{Co} 50$ 50 que dans le Fe-Co 40-60.
4. Déformation plastique. - De nombreuses études ont déjà été faites pour expliquer les variations de la limite élastique $\sigma_{\mathrm{E}}$ des alliages ordonnés du type $\mathrm{L} 2_{0}$ en fonction de l'état d'ordre. Pour rendre compte de ces variations et en particulier du maximum observé sur le $\mathrm{Fe}-\mathrm{Co}-2 \mathrm{~V}$ pour une température légèrement inférieure à la température critique de la transformation ordre-désordre, de nombreux mécanismes ont été proposés dont le plus récent est celui de Moine [27]. Celui-ci, reprenant une idée de Stoloff et Davies [10], considère deux types différents de sources de dislocations :

- les sources constituées de superdislocations ancrées en deux points, et dont le plan de glissement est confondu avec le plan de la paroi d'antiphase ;

- les sources constituées par des dislocations simples ou des dislocations doubles dont le plan de la paroi d'antiphase n'est pas un plan de glissement.

Le calcul des contraintes nécessaires pour débloquer chacun des deux types de sources a été effectué par Moine et al. [15] pour le $\mathrm{Fe}-\mathrm{Co}-2 \mathrm{~V}$.

Expérimentalement, il a été constaté que l'alliage se déforme avant le "pic» de limite élastique, c'est-à-dire pour un degré d'ordre élevé, en mettant en jeu des dislocations doubles, et après le "pic ", en mettant en jeu des dislocations simples. De plus les valeurs expérimentales des contraintes sont en bon accord avec les contraintes calculées.

Pour notre part, nous avons effectué des essais de traction à chaud et après trempe sur le Fe-Co 50-50 et le $\mathrm{Fe}-\mathrm{Co}$ 40-60.

4.1 ESSAIS DE TRACTION A CHAUd. - Sur les figures 4 et 5 , nous avons représenté les variations de la limite élastique $\sigma_{\mathrm{E}}$ et de la charge à la rupture $\sigma_{\mathrm{R}}$ en fonction de la température d'essai pour le $\mathrm{Fe}-\mathrm{Co}-2 \mathrm{~V}$ et pour le $\mathrm{Fe}-\mathrm{Co}$ 40-60. On observe sur ce dernier alliage un pic de limite élastique moins accusé que dans le cas du Fe-Co-2 V, et dont la position correspond à une température plus faible que pour l'alliage ternaire $\left(690^{\circ} \mathrm{C}\right.$ pour le $\mathrm{Fe}-\mathrm{Co}-2 \mathrm{~V}$, $630{ }^{\circ} \mathrm{C}$ pour le $\mathrm{Fe}-\mathrm{Co} 40-60$ ).

Les données relatives au pic de limite élastique des alliages étudiés sont résumées dans le tableau I

TABLEAU I

\section{Essais à chaud}

\begin{tabular}{|c|c|c|c|c|c|}
\hline & \multirow[b]{2}{*}{$T_{\mathrm{c}}{ }^{\circ} \mathrm{C}$} & \multicolumn{2}{|c|}{$\begin{array}{c}\text { Position du pic } \\
\text { de limite } \\
\text { élastique }\end{array}$} & \multicolumn{2}{|c|}{$\begin{array}{c}\text { Distance } \\
\text { d'équilibre } \\
\text { théorique } \\
\text { d'une super- } \\
\text { dislocation }(\AA)\end{array}$} \\
\hline & & $\theta^{\circ} \mathrm{C}$ & $S$ & Coin & Vis \\
\hline & - & - & - & 一 & - \\
\hline $\mathrm{Fe}-\mathrm{Co}-2 \mathrm{~V}$ & 725 & 690 & 0,50 & 550 & 400 \\
\hline $\mathrm{Fe}-\mathrm{Co} 40-60$ & 685 & 630 & 0,65 & 500 & 350 \\
\hline
\end{tabular}

Il apparaît également les valeurs correspondantes de la distance d'équilibre théorique $r_{\mathrm{e}}$ d'une super- 


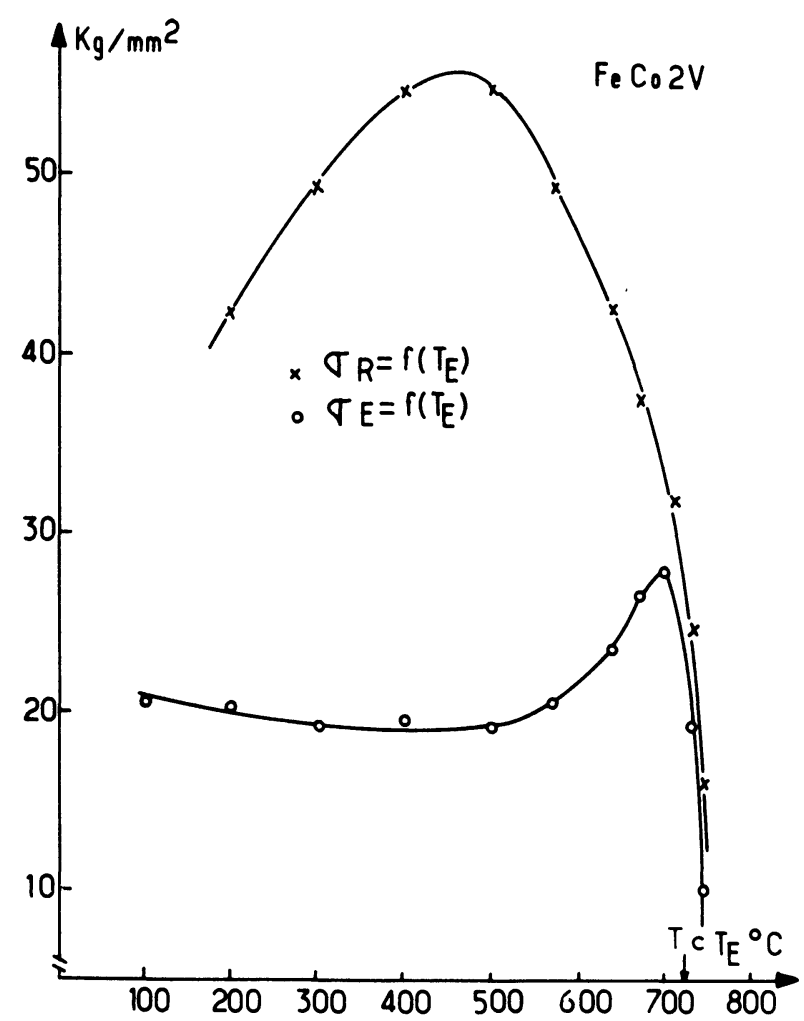

FIG. 4. - Limite élastique $\left(\sigma_{E}\right)$ et charge à la rupture $\left(\sigma_{R}\right)$ en fonction de la température d'essai, alliage $\mathrm{Fe}-\mathrm{Co}-2 \mathrm{~V}$.

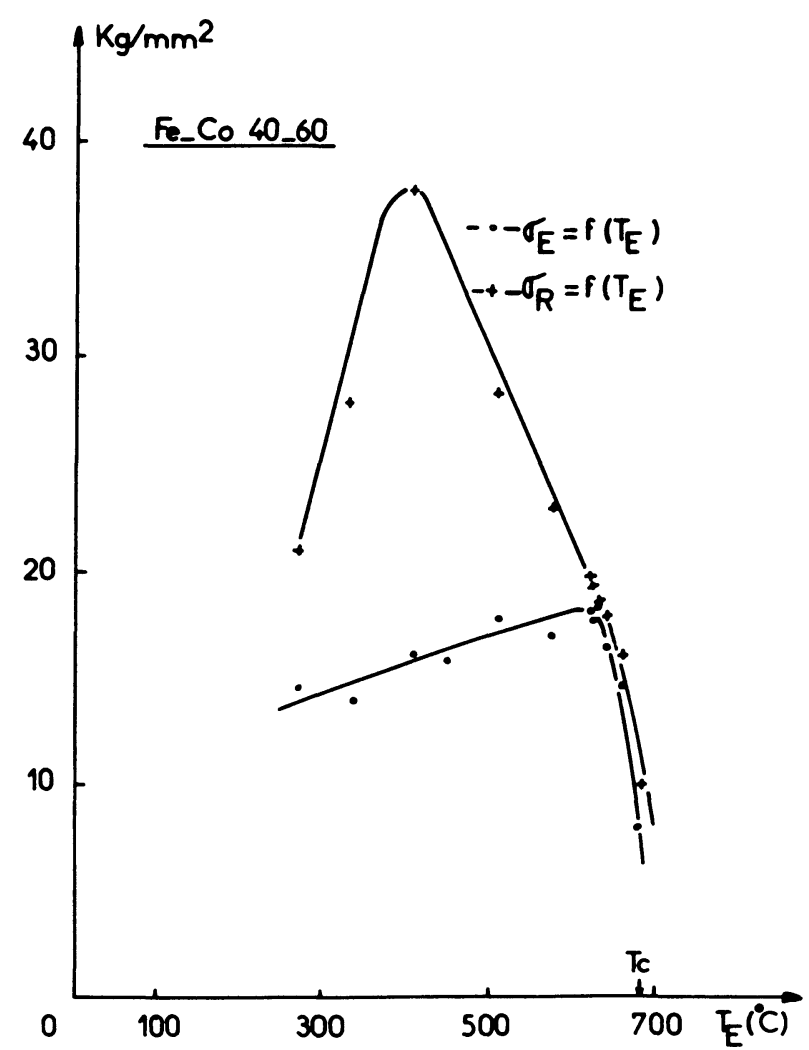

FIG. 5. - Limite élastique $\left(\sigma_{\mathrm{E}}\right)$ et charge à la rupture $\left(\sigma_{\mathrm{R}}\right) \mathrm{e}_{\mathrm{i}}$ fonction de la température d'essai, alliage Fe-Co 40-60.

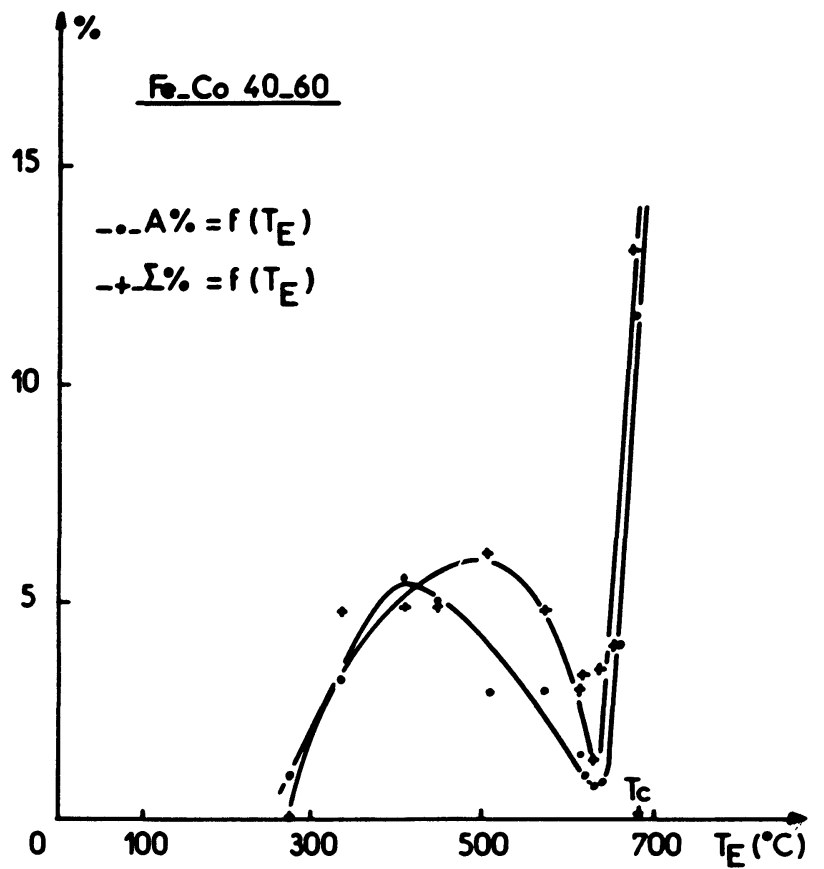

Fig. 6. - Allongement à la rupture $(\mathrm{A} \%)$ et striction $(\Sigma \%)$ en fonction de la température d'essai, alliage Fe-Co 40-60.

dislocation. Nous donnerons le calcul et l'intérêt de $r_{\mathrm{e}}$ plus loin. A la température du pic, l'alliage $\mathrm{Fe}-\mathrm{Co}$ 40-60 passe par un creux de fragilité ; il devient très ductile au-delà. Le même phénomène a été observé sur le Fe-Co-2 V par Dinhut et al. [16].

Nous avons cherché à vérifier si le mécanisme de déformation proposé par Moine s'appliquait au Fe-Co 40-60 et était responsable du " pic ».

L'observation au microscope électronique de la partie utile d'éprouvettes déformées à une température voisine du pic a permis de voir des domaines antiphases d'environ $700 \AA$ (Fig. $7 a$ ), alors que dans une zone non déformée (tête de l'éprouvette) les domaines ont une taille moyenne de $2500 \AA$ (Fig. 7b). Il est raisonnable de penser que les gros domaines thermiques existant à la température de l'essai, ont été traversés par des dislocations simples qui, au cours de la déformation, ont laissé une faute dans leur sillage, l'intersection de ces différentes fautes délimitant de nouveaux petits domaines à l'intérieur des domaines initiaux. Un réarrangement des parois de faute au cours des manipulations ultérieures donne une forme arrondie à ces petits domaines. Dinhut et al. [16] ont observé sur l'alliage Fe-Co-2 V déformé à une température supérieure à celle du pic, des petits domaines délimités par des parois bien rectilignes. En trempant les échantillons de Fe-Co 40-60 déformés à une température inférieure à celle du pic, nous n'avons jamais mis en évidence de ces petits domaines, mais nous avons observé des dislocations doubles (cf. Fig. 8, $T_{\mathrm{E}}=620^{\circ} \mathrm{C}$ ).

4.2 ESSAIS DE TRACTION APRÈS TREMPE. - Les essais de traction après trempe à l'eau nous ont également 


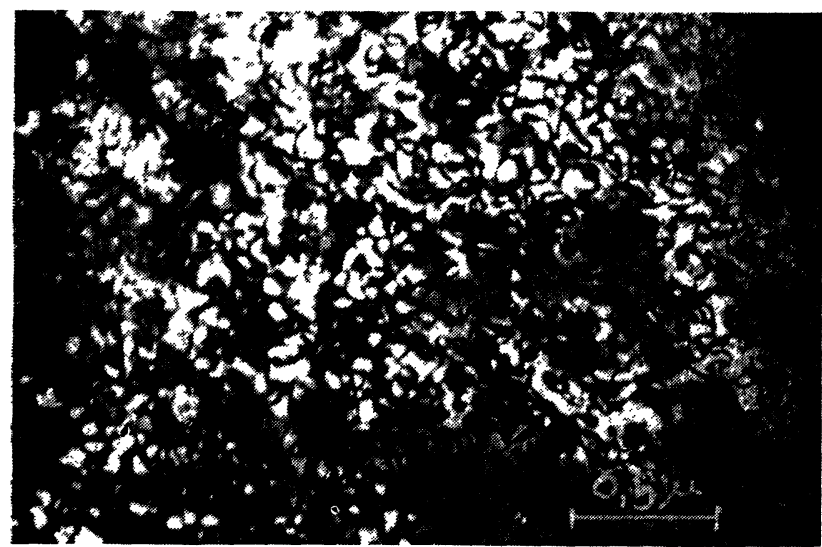

Fig. 7a. - Domaines antiphases, au niveau du pic de limite élastique, dans un échantillon Fe-Co 40-60 $g=(100)(\times 29500)$.

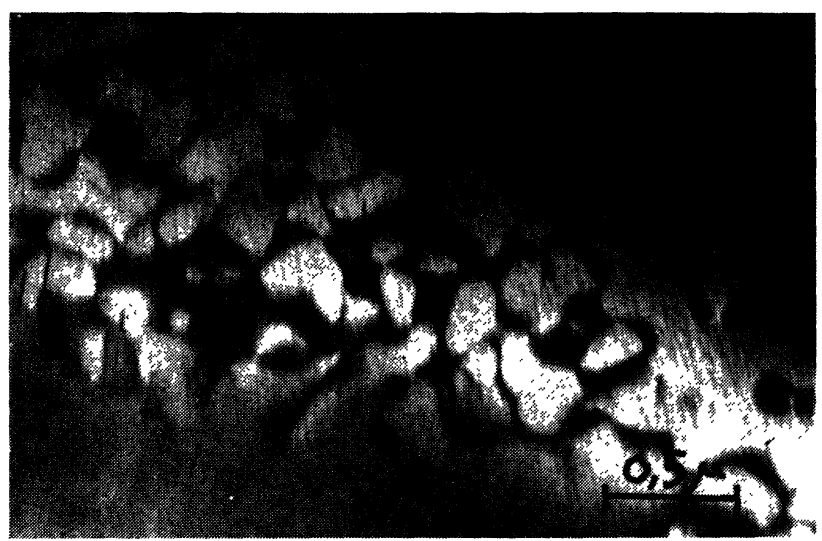

FIG. 7b. - Même échantillon. Zone non déformée $g=(100)$ $(\times 27000)$

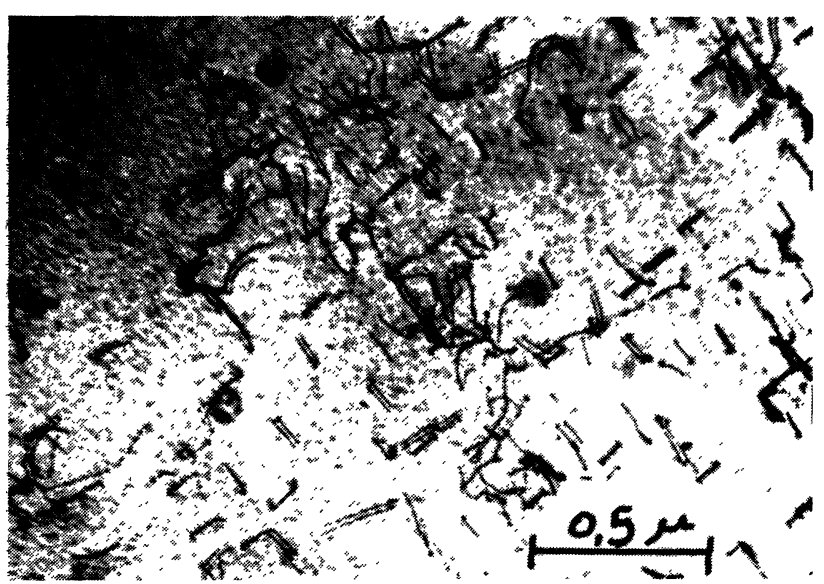

FIG. 8. - Dislocations doubles dans l'alliage Fe-Co 40-60 déformé à $620^{\circ} \mathrm{C}(\times 35000), g=(200)$.

permis de constater un maximum de limite élastique, situé vers $650^{\circ} \mathrm{C}$ pour le $\mathrm{Fe}-\mathrm{Co} 40-60,705^{\circ} \mathrm{C}$ pour pour le $\mathrm{Fe}-\mathrm{Co} 50-50,700{ }^{\circ} \mathrm{C}$ pour le $\mathrm{Fe}-\mathrm{Co}-2 \mathrm{~V}$ (Fig. 9). Ce maximum dépend de la température critique.

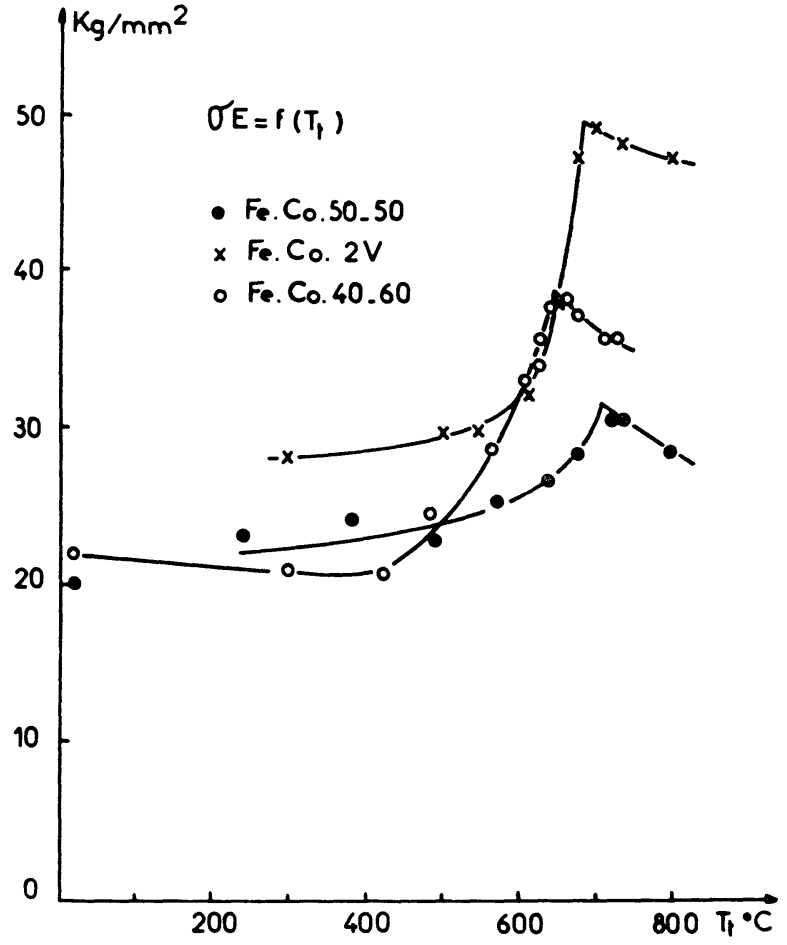

Fig. 9. - Limite élastique en fonction de la température de trempe. Alliages Fe-Co-2 V, Fe-Co 50-50 et Fe-Co 40-60.

TABLEAU II

Essais après trempe

\begin{tabular}{lccccc} 
& & \multicolumn{2}{c}{$\begin{array}{c}\text { Position du pic } \\
\text { de limite }\end{array}$} & \multicolumn{2}{c}{$\begin{array}{c}\text { d'équilibre } \\
\text { théorique } \\
\text { d'une super- } \\
\text { dislocation }(\AA)\end{array}$} \\
& $T_{\mathrm{c}}{ }^{\circ} \mathrm{C}$ & $\theta^{\circ} \mathrm{C}$ & $S$ & $\begin{array}{c}\text { Coin } \\
\text { distique }\end{array}$ & Vis \\
Fe-Co-2 V & -725 & -700 & 0,50 & 650 & 450 \\
Fe-Co 50-50 & 725 & 705 & 0,55 & 700 & 500 \\
Fe-Co 40-60 & 685 & 650 & 0,60 & 600 & 450
\end{tabular}

Nous notons un léger décalage de température observé entre les positions des «pics» obtenus dans les essais à chaud et dans les essais après trempe. De plus, les valeurs de limite élastique sont plus élevées après trempe dans l'état ordonné comme dans l'état désordonné

La figure 10 représente les courbes d'allongement à la rupture et de striction en fonction de la température de trempe pour le $\mathrm{Fe}-\mathrm{Co} 40-60$. On constate un brusque accroissement de $A \%$ et $\Sigma \%$ au niveau du «pic » de limite élastique ; la rupture est alors ductile. Le même phénomène a été observé sur le $\mathrm{Fe}-\mathrm{Co}-2 \mathrm{~V}$, alors que le $\mathrm{Fe}-\mathrm{Co} 50-50$ ne s'allonge que de quelques pour cent, quelle que soit la température de trempe.

4.3 DisCUSSION ET CONCLUSION. - L'étude par traction des propriétés mécaniques des alliages $\mathrm{Fe}-\mathrm{Co}-2 \mathrm{~V}$, Fe-Co 50-50 et Fe-Co 40-60 nous a principalement apporté des résultats dans les domaines suivants : 


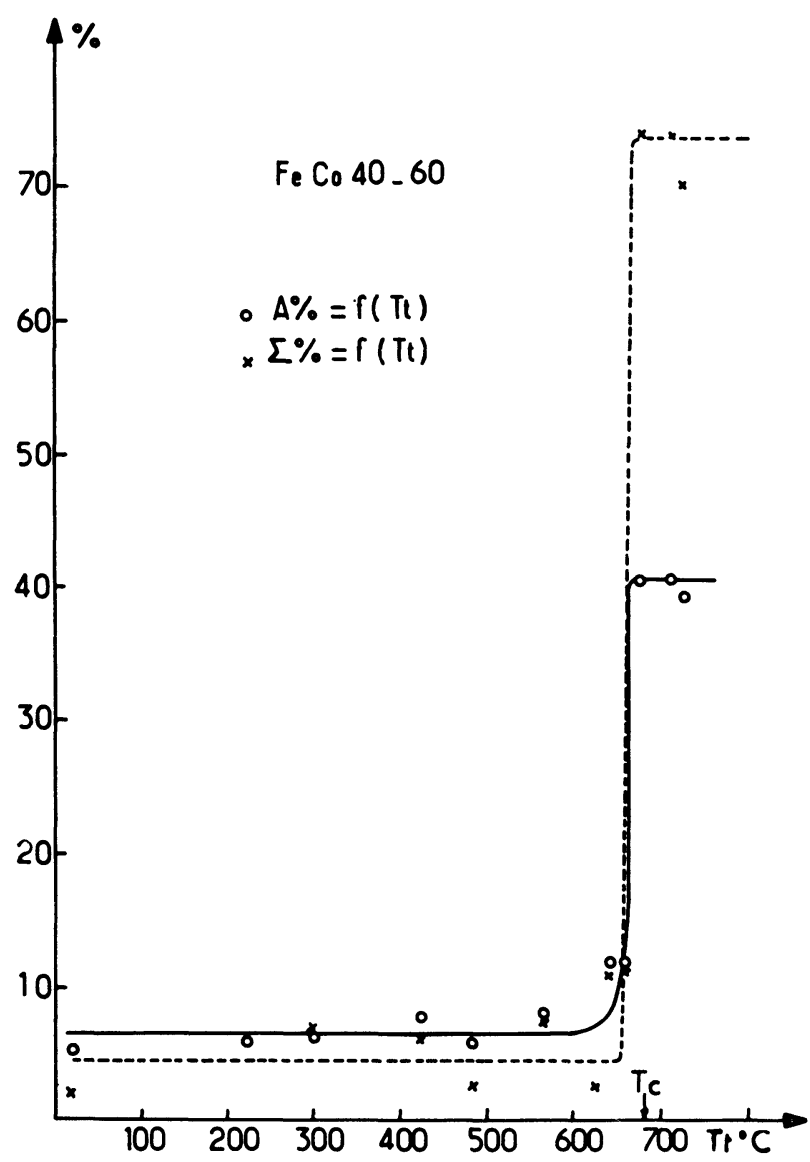

Fig. 10. - Allongement à la rupture (A \%) et striction ( $\Sigma \%$ ) en fonction de la température de trempe. Alliage Fe-Co 40-60.

4.3.1 Limite élastique. - Elle présente dans tous les cas un maximum aussi bien dans les essais «à chaud » que dans les "essais après trempe », pour un degré d'ordre à longue distance $S$ voisin de 0,5 .

Après trempe le vanadium augmente la valeur du maximum $\left(50 \mathrm{~kg} / \mathrm{mm}^{2}\right.$ contre $30 \mathrm{~kg} / \mathrm{mm}^{2}$ pour l'alliage $\mathrm{Fe}-\mathrm{Co}$ 50-50). L'interprétation du maximum de limite élastique [7] à partir d'un changement de mécanisme dans les phénomènes de déformation selon le degré d'ordre des matériaux, reste donc encore valable pour tous ces alliages. Pour des degrés d'ordre $>0,5$ la déformation aurait lieu par dislocations doubles, alors que pour des degrés d'ordre $<0,5$ elle aurait lieu par dislocations simples.

Ce point de vue est confirmé d'une part par l'observation de très petits domaines antiphases ( $\S 4.1$ ) dans les échantillons déformés à chaud à des températures correspondant à un degré d'ordre à longue distance $<0,5$. (L'existence de ces petits domaines peut s'expliquer en effet par le cisaillement des gros domaines thermiques par des dislocations simples); et d'autre part par l'observation de dislocations doubles lorsque le degré d'ordre est $>0,5$ (§ 4.1, Fig. 8).

4.3.2 Fragilité. - Les alliages sont fragiles si $S>0,5$ et ductiles si $S<0,5$. Le caractère de fragilité de l'alliage semble donc bien lié au mode de déformation.

Dans le premier cas $(S>0,5)$ la déformation se produit par dislocations doubles dont la distance d'équilibre $r_{\mathrm{e}}$ des partielles est relativement faible. Les résultats des calculs que nous avons effectués à partir de la théorie de la première approximation (voir annexe et tableaux) montrent que pour tous les alliages étudiés la distance d'équilibre correspondant au «pic» de limite élastique est d'environ $500 \AA$.

Stoloff et Dillamore [29] ont proposé que lorsque la distance d'équilibre des partielles d'une dislocation est comprise entre 50 et $500 \AA$ les dislocations doubles ne peuvent pas effectuer de "cross-slip » ce qui induit une fragilité importante. Par contre dans le $2^{\text {e }}$ cas $(S<0,5)$ chaque partielle se comporte comme une dislocation simple vis-à-vis du glissement dévié. Il s'ensuit que l'alliage devient ductile dans ces conditions puisque le nombre de plans de glissement augmente.

Nous avons donc confirmé sur nos alliages la proposition de Stoloff et Dillamore.

4.3.3 Influence de la non-stochiométrie et $d u$ vanadium. - L'alliage non stœchiométrique a une température de transition fragile-ductile inférieure à celle de l'alliage Fe-Co 50-50 et de l'alliage Fe-Co-2 V. Cela s'explique très bien si l'on remarque que la distance d'équilibre critique $(500 \AA)$ est atteinte pour une température plus faible (cf. Fig. 11).

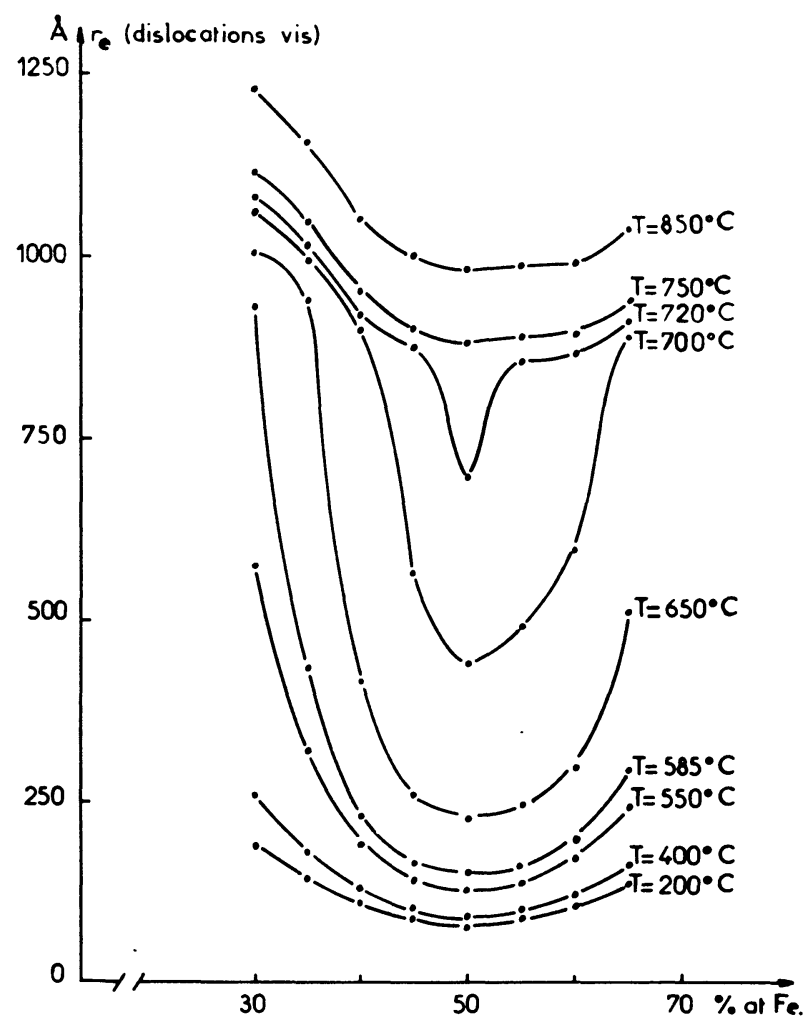

FIG. 11. - Distance d'équilibre $r_{\mathrm{e}}$ des dislocations vis dans un plan (110) en fonction de la concentration en fer. 
L'addition de vanadium contribue à diminuer la fragilité de nos alliages. C'est un résultat semblable à celui observé par Clark [30] dans le laiton $\beta-\mathrm{Cu} 48 \%$ $\mathrm{Zn}$, lorsqu'il ajoute à cet alliage un $3^{\mathrm{e}}$ élément en l'occurrence l'or. D'après cet auteur le $3^{\mathrm{e}}$ élément aurait une influence sur le « cross-slip ».

\section{Annexe}

Energie d'une frontière antiphase.

Distance d'équilibre des 2 partielles d'une superdislocation.

Pour calculer l'énergie d'une frontière antiphase, la méthode utilisée consiste à dénombrer le nombre de paires d'atomes $\mathrm{AA}, \mathrm{BB}$ et $\mathrm{AB}$ premiers voisins dans l'unité de surface du plan de glissement considéré, avant et après glissement d'une dislocation de vecteur de Burgers $\frac{1}{2}<111>$.

Avant glissement, l'énergie du métal peut s'écrire :

$$
E_{0 .}=N_{\mathrm{AA}} \cdot V_{\mathrm{AA}}+N_{\mathrm{BB}} V_{\mathrm{BB}}+N_{\mathrm{AB}} V_{\mathrm{AB}}
$$

où $N_{\mathrm{AA}}$ est le nombre de liaisons $\mathrm{AA}, \ldots$

$V_{\mathrm{AA}}$ est l'énergie d'une liaison AA, ...

Après glissement, celle-ci devient :

$$
\begin{aligned}
E=\left(N_{\mathrm{AA}}+\Delta N_{\mathrm{AA}}\right) V_{\mathrm{AA}}+( & \left.N_{\mathrm{BB}}+\Delta N_{\mathrm{BB}}\right) V_{\mathrm{BB}}+ \\
+ & \left(N_{\mathrm{AB}}+\Delta N_{\mathrm{AB}}\right) V_{\mathrm{AB}} .
\end{aligned}
$$

L'augmentation d'énergie du cristal, égale à l'énergie de la faute créée est :

$$
\begin{aligned}
E-E_{0}=\Gamma=\Delta N_{\mathrm{AA}} V_{\mathrm{AA}} & + \\
& +\Delta N_{\mathrm{BB}} \cdot V_{\mathrm{BB}}+\Delta N_{\mathrm{AB}} V_{\mathrm{AB}} .
\end{aligned}
$$

Or le nombre de liaisons partant d'un atome $\mathrm{A}$, est :

$$
N_{\mathrm{A}} \cdot Z=N_{\mathrm{AA}}+\frac{N_{\mathrm{AB}}}{2}
$$

$N_{\mathrm{A}}:$ nombre d'atomes $\mathrm{A}$

$Z$ : nombre de plus proches voisins

et d'un atome B :

$$
N_{\mathrm{B}} \cdot Z=N_{\mathrm{BB}}+\frac{N_{\mathrm{AB}}}{2} \quad N_{\mathrm{B}}: \text { nombre d'atomes B . }
$$

Ces deux relations permettent d'écrire :

$$
\Delta N_{\mathrm{AA}}=\Delta N_{\mathrm{BB}}=-\frac{\Delta N_{\mathrm{AB}}}{2},
$$

soit :

$$
\Gamma=\Delta N_{\mathrm{AA}}\left(V_{\mathrm{AA}}+V_{\mathrm{BB}}-2 V_{\mathrm{AB}}\right)
$$

ou

$$
\Gamma=2 v \cdot \Delta N_{\mathrm{AA}} .
$$

Cette relation est générale, et dans le cas de $n$ glissements successifs, on a :

$$
\Gamma^{(n)}=2 v \cdot \Delta N_{\mathrm{AA}}^{(n)}
$$

$\Delta N_{\mathrm{AA}}^{(n)}$ est la variation du nombre de liaisons $\mathrm{AA}$ après $n$ glissements ; $v$ est donné par la théorie de la première approximation.

Un calcul de $\Delta N_{\mathrm{AA}}^{(n)}$ dans le cas d'un alliage de type L2 0 stœchiométrique a déjà été effectué par Eymery et al. [28]. Nous l'avons généralisé à la non-stœchiométrie et pour $n=1$, l'énergie d'une frontière antiphase s'écrit :

$$
\begin{array}{r}
\Gamma=2 N Z^{\prime} v \cdot F_{\mathrm{A}}^{2} \times \\
\times F_{\mathrm{B}}^{2} \cdot \sigma+F_{\mathrm{A}} F_{\mathrm{B}} \cdot \sigma^{2}+F_{\mathrm{B}}^{2} \cdot S^{2}+\left(3 F_{\mathrm{A}} F_{\mathrm{B}}-1\right) S^{4}- \\
-\sigma S^{2}-F_{\mathrm{A}}^{2} \sigma^{2} S^{2}+3 F_{\mathrm{A}}^{2} \sigma S^{4}
\end{array}
$$

où $F_{\mathrm{A}}$ et $F_{\mathrm{B}}$ sont les fractions en atome $\mathrm{A}$ et $\mathrm{B} ; S$ et $\sigma$ les paramètres d'ordre à longue et à courte distance ; $N$ le nombre d'atomes par unité de surface du plan de faute, et $Z^{\prime}$ le nombre de liaisons qui partant d'un atome traversent effectivement la surface de faute.

La distance d'équilibre $r_{\mathrm{e}}$ entre deux dislocations de vecteurs de Burgers $\frac{1}{2}<111>$ peut alors être calculée, en égalant la force de répulsion mutuelle entre les deux dislocations à la tension superficielle de la frontière antiphase.

On obtient alors :

$$
r_{e}=\frac{\mu}{2 \pi} \frac{\mathbf{b}^{2}}{\Gamma(h k l)}\left\{\cos ^{2} \theta+\frac{\sin ^{2} \theta}{1-v}\right\}
$$

où $\mu$ est le module de cisaillement,

b est le vecteur de Burgers,

$v$ le coefficient de Poisson,

et $\theta$ l'angle entre la ligne de dislocation et le vecteur de Burgers.

La figure 11 représente les variations de la distance d'équilibre des dislocations vis $(\theta=0)$ dans un plan (110) de l'alliage Fe-Co en fonction de la concentration en fer.

\section{Bibliographie}

[1] Stanley, J. K., Trans. Amer. Soc. Metals 42 (1950) 168.

[2] Facaros, G. et Trap, R. H., Rept. 8-98839-021 - A. Westinghouse Electric Corporation (1959).

[3] Marcinkowski, M. J. et Chessin, H., Phil. Mag. 10 (1964) 837.

[4] Chen, C. W., Cobalt 22 (1964) 1.
[5] Marcinkowski, M. J. et Fisher, R. M., Trans. AIME 233 (1965) 293.

[6] Marcinkowski, M. J. et Larsen, J., Metal. Trans. 1 (1970) 1034.

[7] Sheng-Ti-Fong, Marcinkowski, M. J. et Sadamanda, K., Acta. Met. 21 (1973) 799. 
[8] Chen, C. W. et Wiener, G. W., J. Appl. Phys. 30 (1959) $199 \mathrm{~S}$.

[9] Chen, C. W., J. Appl. Phys. Suppl. 32 (1961) 348 S.

[10] Stoloff, N. S. et Davies, R. G., Acta Met. 12 (1964) 473.

[11] Johnston, T. L., Davies, R. G. et Stoloff, N. S., Phil. Mag. 12 (1965) 305.

[12] Jordan, K. R. et Stoloff, N. S., Trans. Japan Inst. Metals. Suppl. 9 (1968) 281.

[13] Jordan, K. R. et Stoloff, N. S., Trans. M. S. AIME 245 (1969) 2027.

[14] Koylu, Y. G., Hancok, G. F. et Rawlings, R. D., Phys. Stat. Sol. (a) 16 (1973) 73.

[15] Moine, P., Eymery, J. P. et Grosbras, P., Phys. Stat. Sol. (b) 46 (1971) 177.

[16] Dinhut, J. F., Eymery, J. P. et Moine, P., Phys. Stat. Sol. (a) 12 (1972) 153

[17] Bonou, T., Thèse de 3e Cycle. Poitiers (1973).

[18] Eymery, J. P., Grosbras, P. et Moine, P., Revue Phys. Appl. 8 (1973) 139.
[19] Warren, B. E., X-Rays Diffraction (Addison Wesley) 1969, 206.

[20] KraHL, D., Fourth European Regional Conference. Rome (1968) 441.

[21] ENGLish, A. T., Trans AIME 14 (1966) 236.

[22] Hansen, M. et Anderko, P., Binary Alloy Systems (Mc Graw-Hill) 1958.

[23] Ellis, N. C. et Greiner, E. S., Trans. Am. Soc. Metals 29 (1941) 415.

[24] Demenet, J. L., Thèse de 3e Cycle. Poitiers (1973)

[25] Goman'kov, V. I., Litvin, D. F., Loshmanov, A. A., Lyashenko, B. G. et Puzei, I. M., Sov. Phys. Cryst. 7 (1962) 637.

[26] Girifalco, L. A., J. Phys. Chem. Solids 24 (1964) 323.

[27] Morne, P., Thèse d'Etat. Poitiers (1968).

[28] Eymery, J. P., Gaboriaud, R. J., Dinhut, J. F. et Grilhe, J., Scripta Met. 6 (1972) 707.

[29] Stoloff, N. S. et Dillamore, I. L., Ordered Alloys. Proceeding of the 3rd Bolton. Landing Conference AIME (1970) 544.

[30] Clark, H. McI., Phil. Mag. 16 (1967) $85 j$. 